\title{
Synthesis of 2-Methyl 16-Membered Macrolide Derived from Tylosin
}

\author{
Yuichi Terui, Kenji Kinoshita, Yoshie Kaneda, Toshi Akashi, Takuya Hamaguchi, \\ Akira Kawashima
}

Received: October 14, 2005 / Accepted: February 1, 2006

(C) Japan Antibiotics Research Association

\begin{abstract}
To improve the metabolic stability of a 16membered macrolide, 2-methylated derivatives of desmycosin were synthesized. Among these derivatives, $2 \beta$-methyldesmycosin retained antibacterial activity and showed improved stability in rat serum compared to desmycosin.
\end{abstract}

Keywords 16-membered macrolide, tylosin, desmycosin, metabolic stability

\section{Introduction}

Macrolide antibiotics have played important roles as antibacterial agents for half a century, and years of assiduous efforts have been devoted to developing their derivatives more effectively. This research and development has led to the success of second-generation macrolides, such as clarithromycin and azithromycin [1]. While erythromycin and its derivatives have been extensively prescribed, pathogens resistant to these compounds have become increasingly prevalent. To address the problem of resistant bacteria, third-generation macrolides known as "ketolides" were developed, and the first ketolide antibiotic, telithromycin, was recently approved for use [1].

Sixteen-membered macrolide antibiotics have also been used for the treatment of respiratory tract infections. While not as widely prescribed as erythromycin and its

Y. Terui (Corresponding author), Y. Kaneda, T. Akashi, T. Hamaguchi, A. Kawashima: Medicinal Research Laboratories, Taisho Pharmaceutical Co., Ltd., 1-403, Yoshinocho, Kita-ku, Saitama-shi, Saitama 331-9530, Japan,

E-mail: yuichi.terui@po.rd.taisho.co.jp derivatives, these antibiotics are known to have a number of advantages, including better gastrointestinal tolerance, absence of QT increase, and lack of drug-drug interactions. Nevertheless, one of the contributing factors in the low rates of prescription of 16-membered macrolides is their low metabolic stability. In the case of spiramycin, the ringopening derivative was identified as its metabolite by esterase in rat serum [2]. This result was not reported with erythromycin and its derivatives. Our approach to improving the metabolic stability of 16-membered macrolides is to introduce the methyl group at position 2, as with 14-membered macrolides. 2-Methyl 16-membered macrolides were expected to acquire resistance to esterase caused by steric hindrance around the ester bond. Kageyama et al. also reported the synthesis of ( $2 R$ and $2 S$ )3-deoxy-5-O-(4-deoxymycaminosyl)-2-C-methyltylonolide using the same strategy, but no data on the stability of these compounds are available [3]. Here we describe the synthesis of 2-methyldesmycosin and its stability against rat esterase.

\section{Results}

Desmycosin (2), prepared from tylosin (1), was converted to 3 using a previously reported method [4]. The methylation of $\mathbf{3}$ yielded mono- and di-methylated derivatives $(\mathbf{4}, \mathbf{5})$ in the ratio $2: 1$. Contrary to expectations,
K. Kinoshita: Faculty of Pharmaceutical Sciences, Toho University, 2-2-1, Miyama, Funabashi-shi, Chiba 274-8510, Japan. 


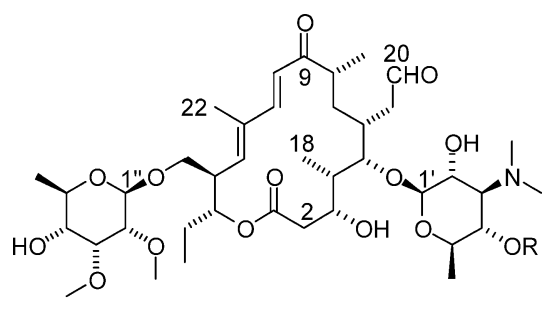

$1: \mathrm{R}=\beta$-mycinosyl
$2: \mathrm{R}=\mathrm{H}$

Fig. 1 Structures of tylosin (1) and desmycosin (2).

4 was derived as a single isomer and no enol form was detected in ${ }^{1} \mathrm{H}$ and ${ }^{13} \mathrm{C}$ NMR. Reduction of $\mathbf{4}$ yielded $\mathbf{6}$, subsequently only the 9-hydroxyl group was oxidized by Dess-Martin oxidation to produce 7 . The stereochemistry of 6 at positions 2 and 3 was determined by analyzing the coupling constants and NOESY spectrum. The coupling constant between $2-\mathrm{H}$ and $3-\mathrm{H}$ was $2.4 \mathrm{~Hz}$, indicating gauche orientation. And that between $3-\mathrm{H}$ and $4-\mathrm{H}$ was $10.7 \mathrm{~Hz}$, indicating anti orientation. The multiplicity of $3-\mathrm{H}$ could be measured by adding $\mathrm{D}_{2} \mathrm{O}$. Together with the following NOEs, these findings revealed the stereochemistry of $\mathbf{6}$ at positions $\mathbf{2}$ and $\mathbf{3}$ as shown in Fig. 2: 2-H/18-H, 2-Me/3-H, 3-H/5-H and 3-H/1'-H. 3-epi configuration was also supported by Furuuchi's data [5]. Recently they reported on the synthesis of 3-epileucomycin $\mathrm{A}_{7}$ (3-epi-LM-A $)$ via reduction of 3-keto-LM$\mathrm{A}_{7}$.

Initially we planned to produce several stereoisomers of 2-methyldesmycosin by the above route, unfortunately, however, methylation and the reduction process progressed stereoselectively. Furuuchi [5] also reported that the stability of 3-epi-LM-A $\mathrm{A}_{7}$ is significantly reduced compared to $\mathrm{LM}-\mathrm{A}_{7}$. Accordingly, we planned to synthesize other isomers of 2-methyldesmycosin.

$2 \beta$-Methyldesmycosin (14) and $2 \alpha$-methyldesmycosin (15) were synthesized as shown in Scheme 1. 1 was converted to protected compound $\mathbf{8}$ using a previously reported method [4]. Reduction of the 9-keto group of $\mathbf{8}$ yielded 9 as a single isomer. After deprotection of the formyl group of $\mathbf{9}$, the 3-hydroxyl and 20-formyl groups were protected as 3,20-(O-silyl)acetal together with a 9hydroxyl group as silyl ether to produce 10. Three acetyl groups at sugar moieties were converted to triethylsilyl groups, following methylation in the presence of 1,3dimethyl-3,4,5,6-tetrahydro-2(1H)-pyrimidinone (DMPU) yielded 12 and 13 in the ratio $10: 1.12$ and 13, separated by silica gel pTLC, were both deprotected to yield 14 and 15. The stereochemistry of $\mathbf{1 4}$ and $\mathbf{1 5}$ were determined

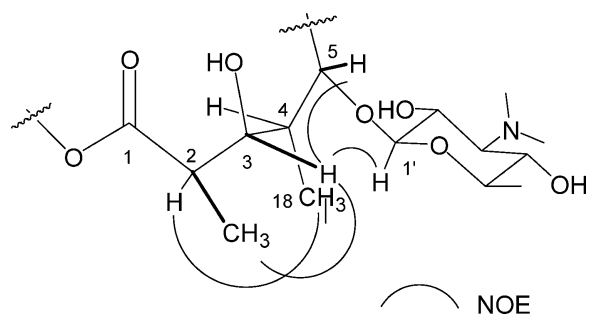

Fig. 2 Stereo-drawing of $\mathbf{6}$ at positions 2 and 3.

through NOESY spectrum analysis. Correlation between $2-\mathrm{H}$ and $22-\mathrm{H}$ of $\mathbf{1 4}$ revealed a $\beta$ configuration of the 2methyl group. In contrast, a correlation was seen between 2-Me and 22-H of $\mathbf{1 5 .}$

The in vitro antibacterial activities of 7, 14, and 15 are summarized in Table 1.7 and $\mathbf{1 5}$ displayed poor activity compared to the parent macrolide desmycosin (2). 2-Methyl3-keto-desmycosin, the deprotected compound of 4 , and 2,2-dimethyl-3-keto-desmycosin, the deprotected compound of 5, also displayed poor activity (data not shown). Only 14 retained antibacterial activity approximately equivalent to that of desmycosin (2).

\section{Discussion}

In the antibacterial test of desmycosin derivatives, only $\mathbf{1 4}$ showed activity approximately equivalent to that of the parent compound desmycosin (2). 14 features the same stereochemistry as erythromycin at positions 2 and 3 , indicating the importance of this stereochemistry.

The stability of $\mathbf{2}$ and $\mathbf{1 4}$ in rat serum was estimated by LC/MS. In the case of desmycosin (2), the peak corresponding to the ring-opening metabolite (MW 790) was observed after 1-hour incubation in rat serum (Fig. 3b). No ring-opening metabolites were detected after incubation of desmycosin (2) in a phosphate buffer (Fig. 3d) and $\mathbf{1 4}$ in rat serum (Fig. 3f). These results suggested that 14 acquired resistance to esterase in rat serum due to the steric hindrance of the 2-methyl group. To verify this data, we plan to confirm the structure of the desmycosin metabolite (Fig. 3b) and to prepare 14 using the combinatorial biosynthesis method for the in vivo antibacterial test. These results will be reported elsewhere.

\section{Experimental}

\section{General Procedure}

${ }^{1} \mathrm{H}$ and ${ }^{13} \mathrm{C}$ NMR spectra were measured on a JEOL Alpha 


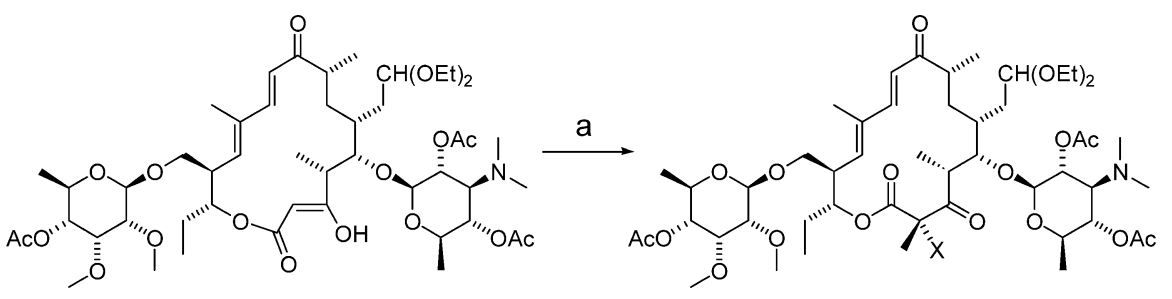

3

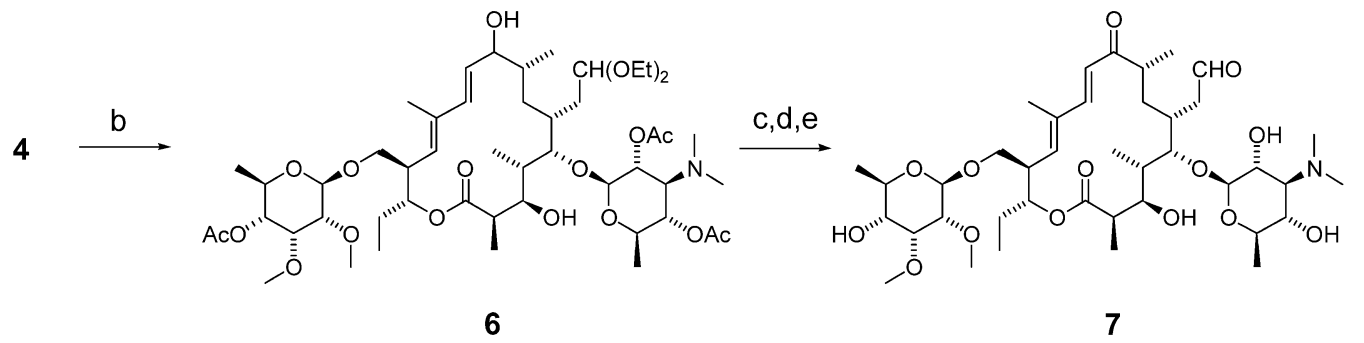

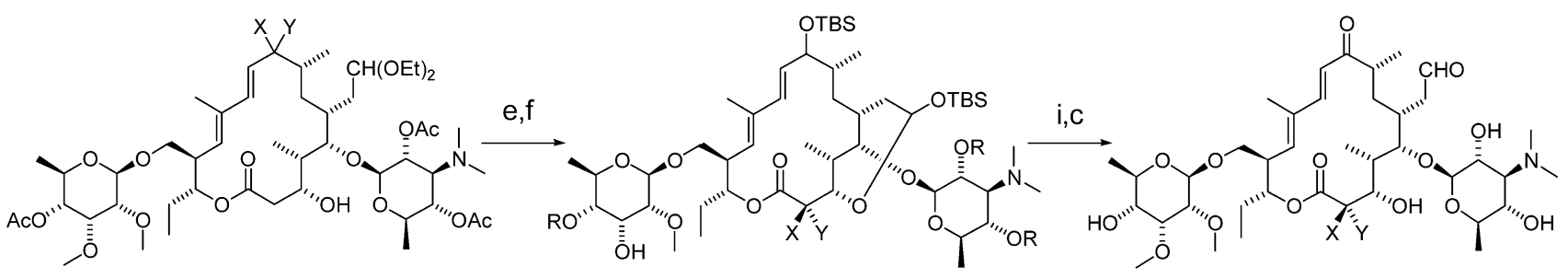

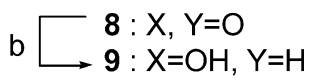

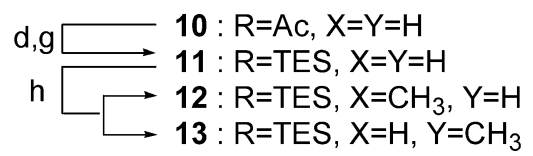

Reagents and conditions: (a) $\mathrm{CH}_{3} \mathrm{l}, \mathrm{tBuOK}, \mathrm{THF}, 0^{\circ} \mathrm{C}, 2$ hours; (b) $\mathrm{CeCl}_{3}-7 \mathrm{H}_{2} \mathrm{O}, \mathrm{NaBH}_{4}, \mathrm{MeOH}^{\circ} 0^{\circ} \mathrm{C}, 30$ minutes; (c) Dess-Matin

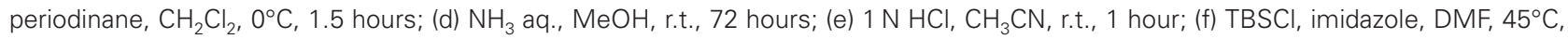
18 hours; (g) TESCl, imidazole, DMF, r.t., 18 hours; (h) LDA, $\mathrm{CH}_{3} \mathrm{l}$, THF-DMPU $2: 1,-78^{\circ} \mathrm{C}$, 1 hour; (i) $\mathrm{F}_{2} \mathrm{CHCOOH}, \mathrm{CH}_{3} \mathrm{CN}-\mathrm{H}_{2} \mathrm{O} 1: 1$, $45^{\circ} \mathrm{C}, 72$ hours.

\section{Scheme 1}

Table 1 In vitro antibacterial activities of desmycosin derivatives

\begin{tabular}{|c|c|c|c|c|c|c|c|}
\hline \multirow{2}{*}{ Strain } & \multirow{2}{*}{ Characteristics } & \multicolumn{6}{|c|}{$\mathrm{MIC}(\mu \mathrm{g} / \mathrm{ml})$} \\
\hline & & 2 & 7 & 14 & 15 & CAM & RKM \\
\hline Streptococcus pneumoniae IID553 & & 0.5 & 2 & 0.5 & 2 & 0.03 & 0.12 \\
\hline S. pneumoniae 205 & ermB $B^{+}$ & $>128$ & $>128$ & $>128$ & 32 & $>128$ & 32 \\
\hline S. pneumoniae 210 & $\operatorname{mefA}^{+}$ & 2 & 4 & 2 & 4 & 4 & 0.25 \\
\hline Haemophilus influenzae ATCС33533 & & 4 & 16 & 8 & 32 & 16 & 8 \\
\hline
\end{tabular}

CAM : clarithromycin, RKM : rokitamycin

500 or JEOL Lambda 500 spectrometer. Chemical shifts are reported in parts per million (ppm) with TMS as an internal standard. Coupling constants $(J)$ are given in hertz
(Hz). Optical rotations were measured on a JASCO DIP360 digital polarimeter. Mass spectra were recorded on a Micromass Platform LC or a Micromass Q-Tof 2. LC/MS 


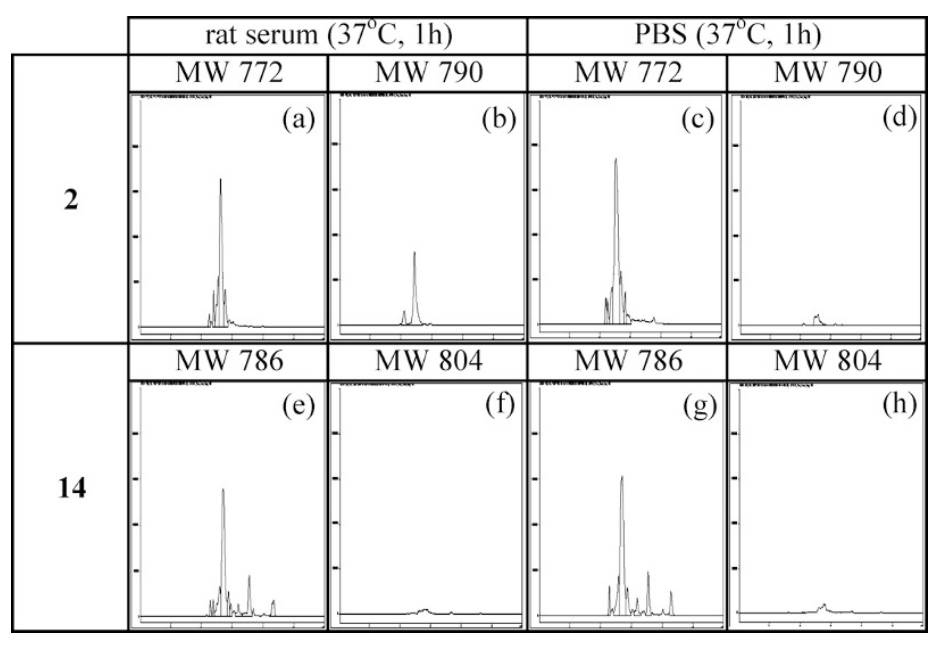

Fig. $3 \mathrm{LC} / \mathrm{MS}$ analysis of $\mathbf{2}$ and $\mathbf{1 4}$ after incubation in rat serum or PBS for 1 hour at $37^{\circ} \mathrm{C}^{\mathrm{a}}$.

${ }^{a}$ Each compound was detected by expected molecular weight and all data are written in same scale.

spectra were obtained on an Agilent 1100 LC/MSD SL system.

\section{Antibacterial Activity Test}

Minimum inhibitory concentration (MIC) was determined by the agar dilution method as recommended by NCCLS [6].

\section{Stability Test in Rat Serum}

Test compound was dissolved in DMSO $(100 \mathrm{mg} / \mathrm{ml})$. The DMSO solution $(10 \mu \mathrm{l})$ was added to EDTA-treated rat serum $(0.5 \mathrm{ml}$, prepared from Wister Rat, 5 weeks of age) and incubated for 1 hour at $37^{\circ} \mathrm{C} . \mathrm{CH}_{3} \mathrm{CN}(0.5 \mathrm{ml})$ was added to the reaction mixture and centrifuged for 5 minutes at $10000 \mathrm{rpm}$. The supernatant was collected and evaporated under reduced pressure. The resulting residue was dissolved to $50 \% \mathrm{EtOH}$ and analyzed by LC/MS. LC/MS conditions are as follows; ionization method: electron spray ionization (ESI); column: YMCPack ODS-AM $100 \times 4.6 \mathrm{~mm}$ I.D.; solvent system: $10 \mathrm{mM} \mathrm{HCOOH}, \mathrm{HCOONH} \mathrm{H}_{4} / \mathrm{CH}_{3} \mathrm{CN}$ gradient; flow rate: $1.0 \mathrm{ml} /$ minute; temperature: $40^{\circ} \mathrm{C}$.

\section{$2 \beta$-Methyl-3-dehydro-2', $4^{\prime}, 4^{\prime \prime}$-tri- $O$-acetyldesmycosin-} 20-diethylacetal (4), 2,2-Dimethyl-3-dehydro-2', 4', 4'"-tri$O$-acetyldesmycosin-20-diethylacetal (5)

2,3-Dehydro-2', 4', 4' -tri- $O$-acetyldesmycosin-20-diethylacetal $(3,1.54 \mathrm{~g}, 1.59 \mathrm{mmol})$, prepared by literature-based procedures [4] from tylosin tartrate (Wako Pure Chemical Industries, Ltd.), was dissolved in THF $(75 \mathrm{ml})$. To this solution, potassium tert-butoxide $(210.9 \mathrm{mg}, 1.88 \mathrm{mmol})$ and methyl iodide $(0.2 \mathrm{ml}, 3.21 \mathrm{mmol})$ were added at $0^{\circ} \mathrm{C}$.
After stirring for 2 hours, the reaction solution was added to water and extracted with EtOAc. The organic solution was dried over anhydrous $\mathrm{MgSO}_{4}$, filtered and evaporated. The resulting residue was chromatographed on silica gel eluting with $50 \%$ EtOAc in hexane to yield $4(595.3 \mathrm{mg}$, $38.1 \%$ yield) and 5 (319.9 mg, 20.2\% yield). 4: ESI-MS $m / z 984(\mathrm{M}+\mathrm{H})^{+} ;[\alpha]_{\mathrm{D}}^{25}=+4.6^{\circ}\left(c 1.05, \mathrm{CHCl}_{3}\right) ; \mathrm{IR} v_{\max }$ $(\mathrm{KBr}) \mathrm{cm}^{-1} 3464,2978,2939,1750,1666,1456,1375$, $1232,1171,1089,1056 ;{ }^{1} \mathrm{H}$ NMR $\left(500 \mathrm{MHz}, \mathrm{CDCl}_{3}\right) \delta$ $0.89(3 \mathrm{H}, \mathrm{t}, J=7.3 \mathrm{~Hz} ; 17-\mathrm{H}), 1.11(3 \mathrm{H}, \mathrm{d}, J=6.7 \mathrm{~Hz} ; 21-$ $\mathrm{H}), 1.31(3 \mathrm{H}, \mathrm{d}, J=7.0 \mathrm{~Hz} ; 2-\mathrm{Me}), 1.80(3 \mathrm{H}, \mathrm{s} ; 22-\mathrm{H}), 1.94$ (3H, s; OAc), 2.03 (3H, s; OAc), 2.08 (3H, s; OAc), 2.30 $\left(6 \mathrm{H}, \mathrm{s} ; 3^{\prime}-\mathrm{NMe}_{2}\right), 3.40\left(3 \mathrm{H}, \mathrm{s} ; 2^{\prime \prime}-\mathrm{OMe}\right), 3.49$ (3H, s; 3"OMe), 3.55 (1H, q, $J=7.3 \mathrm{~Hz}$; 2-H), 5.79 (1H, br d; 13-H), $6.08(1 \mathrm{H}, \mathrm{brd} ; 10-\mathrm{H}), 7.06(1 \mathrm{H}, \mathrm{d}, J=16.5 \mathrm{~Hz} ; 11-\mathrm{H}) ;{ }^{13} \mathrm{C}$ NMR (125 MHz, $\left.\mathrm{CDCl}_{3}\right) \delta 170.6(\mathrm{C}-1), 49.5(\mathrm{C}-2), 13.8$ (2-Me), 209.0 (C-3). 5: ESI-MS m/z $998(\mathrm{M}+\mathrm{H})^{+}$; $[\alpha]_{\mathrm{D}}=-31.1^{\circ}\left(c 1.03, \mathrm{CHCl}_{3}\right)$; IR $v_{\max }(\mathrm{KBr}) \mathrm{cm}^{-1} 3444$, 2979, 2939, 1752, 1723, 1667, 1456, 1374, 1232, 1169, $1152,1090,1056 ;{ }^{1} \mathrm{H}$ NMR $\left(500 \mathrm{MHz}, \mathrm{CDCl}_{3}\right) \delta 0.92(3 \mathrm{H}$, t, $J=7.3 \mathrm{~Hz} ; 17-\mathrm{H}), 0.97(3 \mathrm{H}, \mathrm{d}, J=7.0 \mathrm{~Hz} ; 18-\mathrm{H}), 1.13$ $(3 \mathrm{H}, \mathrm{d}, J=6.7 \mathrm{~Hz} ; 21-\mathrm{H}), 1.33\left(6 \mathrm{H}, \mathrm{s} ; 2-\mathrm{Me}_{2}\right), 1.81(3 \mathrm{H}, \mathrm{s}$; 22-H), 1.95 (3H, s; OAc), 2.05 (3H, s; OAc), $2.11(3 \mathrm{H}, \mathrm{s}$; $\mathrm{OAc}), 2.34\left(6 \mathrm{H}, \mathrm{s} ; 3^{\prime}-\mathrm{NMe}_{2}\right), 3.42\left(3 \mathrm{H}, \mathrm{s} ; 2^{\prime \prime}-\mathrm{OMe}\right), 3.51$ (3H, s; 3"-OMe), $5.94(1 \mathrm{H}, \mathrm{d}, J=11.0 \mathrm{~Hz} ; 13-\mathrm{H}), 6.08(1 \mathrm{H}$, d, $J=16.5 \mathrm{~Hz} ; \mathrm{H}-10), 7.18(1 \mathrm{H}, \mathrm{d}, J=16.5 \mathrm{~Hz} ; 11-\mathrm{H}) ;{ }^{13} \mathrm{C}$ NMR (125 MHz, $\left.\mathrm{CDCl}_{3}\right) \delta 173.4(\mathrm{C}-1), 55.8(\mathrm{C}-2), 21.6$ (2-Me), 22.6 (2-Me), 213.6 (C-3).

2 $\beta$-Methyl-3-epi-9-hydro-2', $4^{\prime}, 4^{\prime \prime}$-tri-Oacetyldesmycosin-20-diethylacetal (6)

To a solution of $4(261.4 \mathrm{mg}, 0.266 \mathrm{mmol})$ in methanol 
$(6.0 \mathrm{ml})$, cerium chloride heptahydrate $(148.4 \mathrm{mg}, 0.398$ $\mathrm{mmol})$ was added. After stirring for 30 minutes at room temperature, sodium borohydride $(400.0 \mathrm{mg}, 10.6 \mathrm{mmol})$ was added slowly at $0^{\circ} \mathrm{C}$. After stirring for 30 minutes, $1 \mathrm{M}$ $\mathrm{Na}_{2} \mathrm{HPO}_{4}(50 \mathrm{ml})$ was added and the mixture was extracted with EtOAc. The organic solution was dried over anhydrous $\mathrm{MgSO}_{4}$, filtered and evaporated. The resulting residue was chromatographed on silica gel eluting with 50\% EtOAc in hexane to yield 6 ( $98.7 \mathrm{mg}, 37.6 \%$ yield). 6: ESI-MS $\mathrm{m} / \mathrm{z}$ $988(\mathrm{M}+\mathrm{H})^{+} ;[\alpha]_{\mathrm{D}}^{25}=+7.0^{\circ}\left(c 1.14, \mathrm{CHCl}_{3}\right) ; \mathrm{IR} v_{\max }(\mathrm{KBr})$ $\mathrm{cm}^{-1}$ 3492, 2977, 2938, 1751, 1708, 1457, 1375, 1232, $1173,1090,1054 ;{ }^{1} \mathrm{H}$ NMR $\left(500 \mathrm{MHz}, \mathrm{CDCl}_{3}\right) \delta 0.72(3 \mathrm{H}$, d, $J=6.7 \mathrm{~Hz} ; 18-\mathrm{H}), 0.88(3 \mathrm{H}, \mathrm{t}, J=7.3 \mathrm{~Hz} ; 17-\mathrm{H}), 0.97$ $(3 \mathrm{H}, \mathrm{d}, J=7.3 \mathrm{~Hz} ; 21-\mathrm{H}), 1.36(3 \mathrm{H}, \mathrm{d}, J=7.3 \mathrm{~Hz} ; 2-\mathrm{Me})$, $1.70(3 \mathrm{H}, \mathrm{s} ; 22-\mathrm{H}), 1.96(3 \mathrm{H}, \mathrm{s} ; \mathrm{OAc}), 2.01$ (3H, s; OAc), $2.08(3 \mathrm{H}, \mathrm{s} ; \mathrm{OAc}), 2.30\left(6 \mathrm{H}, \mathrm{s} ; 3^{\prime}-\mathrm{NMe}_{2}\right), 2.65(1 \mathrm{H}, \mathrm{dq}$, $J=1.8,7.3 \mathrm{~Hz} ; 2-\mathrm{H}), 3.08$ (1H, brt, $J=11.0 \mathrm{~Hz} ; 3-\mathrm{H}), 3.44$ (3H, s; 2"-OMe), 3.49 (3H, s; 3"-OMe), 4.11 (1H, br; 9-H), $5.36(1 \mathrm{H}, \mathrm{d}, J=10.4 \mathrm{~Hz} ; 13-\mathrm{H}), 5.67(1 \mathrm{H}, \mathrm{dd}, J=3.7$, $15.9 \mathrm{~Hz} ; 10-\mathrm{H}), 6.32(1 \mathrm{H}, \mathrm{dd}, J=1.2,15.9 \mathrm{~Hz} ; 11-\mathrm{H}) ;{ }^{13} \mathrm{C}$ NMR (125 MHz, $\left.\mathrm{CDCl}_{3}\right) \delta 177.2$ (C-1), 39.8 (C-2), 17.2 (2-Me), 76.7 (C-3), 76.8 (C-9).

\section{2 $\beta$-Methyl-3-epi-desmycosin (7)}

To a solution of $6(158.8 \mathrm{mg}, 0.161 \mathrm{mmol})$ in $\mathrm{CH}_{2} \mathrm{Cl}_{2}$ $(10 \mathrm{ml})$, Dess-Martin periodinane $(81.8 \mathrm{mg}, 0.193 \mathrm{mmol})$ was added at $0^{\circ} \mathrm{C}$. After stirring for 1.5 hours, $0.2 \mathrm{~N} \mathrm{NaOH}$ aq. was added to the reaction mixture and it was extracted with EtOAc. The organic solution was dried over anhydrous $\mathrm{MgSO}_{4}$, filtered and evaporated. The resulting residue was dissolved in methanol $(10 \mathrm{ml})$. After $28 \% \mathrm{NH}_{3}$ aq. $(0.06 \mathrm{ml})$ was added to this solution, the reaction mixture was stirred for 3 days at room temperature. The solution was evaporated and the resulting residue was dissolved in $\mathrm{CH}_{3} \mathrm{CN}(2.0 \mathrm{ml})$. To this solution, $1 \mathrm{~N} \mathrm{HCl}$ aq. $(0.5 \mathrm{ml})$ was added and the reaction mixture was stirred for 1 hour at room temperature. The solution was evaporated and the resulting residue was purified by silica gel pTLC $\left(\mathrm{CHCl}_{3}\right.$ $\mathrm{MeOH} 4: 1)$ to yield $7(101.0 \mathrm{mg}, 80.1 \%$ yield over three steps). 7: HRTOF-MS $m / z 784.4495(\mathrm{M}-\mathrm{H})^{-}$(calcd for $^{\circ}$ $\left.\mathrm{C}_{40} \mathrm{H}_{66} \mathrm{NO}_{14}: 784.4483\right) ;[\alpha]_{\mathrm{D}}^{25}=-9.2^{\circ}\left(c 0.95, \mathrm{CHCl}_{3}\right)$; IR $v_{\max }(\mathrm{KBr}) \mathrm{cm}^{-1} 3447,2975,2935,1721,1660,1632$, $1598,1458,1380,1271,1169,1084,1064 ;{ }^{1} \mathrm{H}$ NMR $\left(500 \mathrm{MHz}, \mathrm{CDCl}_{3}\right) \delta 0.84(3 \mathrm{H}, \mathrm{d}, J=6.7 \mathrm{~Hz} ; 18-\mathrm{H}), 0.87$ $(3 \mathrm{H}, \mathrm{t}, J=7.3 \mathrm{~Hz} ; 17-\mathrm{H}), 1.13(3 \mathrm{H}, \mathrm{d}, J=6.7 \mathrm{~Hz} ; 21-\mathrm{H})$, 1.19 (3H, d, $\left.J=6.4 \mathrm{~Hz} ; 6^{\prime \prime}-\mathrm{H}\right), 1.20\left(3 \mathrm{H}, \mathrm{d}, J=6.0 \mathrm{~Hz} ; 6^{\prime}-\mathrm{H}\right)$, $1.31(3 \mathrm{H}, \mathrm{d}, J=7.3 \mathrm{~Hz} ; 2-\mathrm{Me}), 1.73(3 \mathrm{H}, \mathrm{s} ; 22-\mathrm{H}), 2.44$ (6H, s; 3'- $\left.\mathrm{NMe}_{2}\right), 2.56(1 \mathrm{H}, \mathrm{qd}, J=7.3,2.1 \mathrm{~Hz} ; 2-\mathrm{H}), 3.15$ (1H, br; 3-H), 3.41 (3H, s; 2"-OMe), 3.54 (3H, s; 3"-OMe), $4.13\left(1 \mathrm{H}, \mathrm{d}, J=7.3 \mathrm{~Hz} ; 1^{\prime}-\mathrm{H}\right), 4.33(1 \mathrm{H}, \mathrm{br} \mathrm{s} ; 5-\mathrm{H}), 4.46$ $\left(1 \mathrm{H}, \mathrm{d}, J=7.6 \mathrm{~Hz} ; 1^{\prime \prime}-\mathrm{H}\right), 4.99$ (1H, td, $J=9.8,2.7 \mathrm{~Hz} ; 15-$
H), $5.85(1 \mathrm{H}, \mathrm{d}, J=10.4 \mathrm{~Hz} ; 13-\mathrm{H}), 6.16(1 \mathrm{H}, \mathrm{d}, J=15.9 \mathrm{~Hz}$; 10-H), 7.02 (1H, d, $J=15.9 \mathrm{~Hz} ; 11-\mathrm{H}), 9.67(1 \mathrm{H}, \mathrm{s} ; 20-\mathrm{H})$; ${ }^{13} \mathrm{C}$ NMR $\left(125 \mathrm{MHz}, \mathrm{CDCl}_{3}\right) \delta 177.1$ (C-1), 40.3 (C-2), 17.7 (2-Me), 77.3 (C-3), 43.9 (C-4), 205.0 (C-9), 202.4 (C20).

\section{9-Hydro-2', 4', 4"-tri-O-acetyldesmycosin-20- diethylacetal (9)}

$2^{\prime}, 4^{\prime}, 4^{\prime \prime}$-Tri- $O$-acetyldesmycosin-20-diethylacetal $(8,50.1 \mathrm{~g})$, prepared by literature-based procedures [4] from tylosin tartrate, was dissolved in methanol (1 liter). To this solution, cerium chloride heptahydrate $(28.8 \mathrm{~g}, 0.077 \mathrm{~mol})$ was added. After stirring at room temperature for 30 minutes, sodium borohydride $(9.7 \mathrm{~g}, 0.26 \mathrm{~mol})$ was added slowly at $0^{\circ} \mathrm{C}$. After stirring for 30 minutes, $1 \mathrm{M} \mathrm{Na}_{2} \mathrm{HPO}_{4}$ $(500 \mathrm{ml})$ was added and the mixture was extracted with EtOAc. The organic solution was dried over anhydrous $\mathrm{MgSO}_{4}$, filtered and evaporated. The resulting residue was chromatographed on silica gel eluting with $60 \%$ EtOAc in hexane to yield $9(22.4 \mathrm{~g}, 49.0 \%$ yield over four steps from tylosin tartrate). 9: ESI-MS $\mathrm{m} / \mathrm{z} 974(\mathrm{M}+\mathrm{H})^{+}$; $[\alpha]_{\mathrm{D}}^{25}=+6.2^{\circ}\left(c 1.06, \mathrm{CHCl}_{3}\right)$; IR $v_{\max }(\mathrm{KBr}) \mathrm{cm}^{-1} 3510$, 2978, 2938, 1750, 1714, 1456, 1374, 1232, 1173, 1090, $1051 ;{ }^{1} \mathrm{H}$ NMR $\left(500 \mathrm{MHz}, \mathrm{CDCl}_{3}\right) \delta 0.90(3 \mathrm{H}, \mathrm{t}$, $J=7.3 \mathrm{~Hz} ; 17-\mathrm{H}), 1.69$ (3H, s; 22-H), 2.00 (3H, s; OAc), $2.02(3 \mathrm{H}, \mathrm{s} ; \mathrm{OAc}), 2.08(3 \mathrm{H}, \mathrm{s} ; \mathrm{OAc}), 2.30\left(6 \mathrm{H}, \mathrm{s} ; 3^{\prime}-\right.$ $\left.\mathrm{NMe}_{2}\right) 3.45$ (3H, s; 2"-OMe), 3.49 (3H, s; 3"-OMe), 4.91 $(1 \mathrm{H}, \mathrm{dd}, J=10.1,2.4 \mathrm{~Hz} ; 9-\mathrm{H}), 5.29(1 \mathrm{H}$, br d, $J=9.8 \mathrm{~Hz}$; 13-H), $5.77(1 \mathrm{H}$, br; $10-\mathrm{H}), 6.41(1 \mathrm{H}, \mathrm{dd}, J=15.8,1.5 \mathrm{~Hz}$; $11-\mathrm{H}) ;{ }^{13} \mathrm{C}$ NMR (125 MHz, $\left.\mathrm{CDCl}_{3}\right) \delta 76.0$ (C-9).

\section{9-Hydro-9,20-di-O-tert-butyldimethylsilyl-2', $4^{\prime}, 4^{\prime \prime}$-tri- $O$ - acetyldesmycosin-3,20-acetal (10)}

The formyl group of 9 (22.2 g, $22.8 \mathrm{mmol})$ was deprotected as mentioned above and the resulting residue was dissolved in DMF $(250 \mathrm{ml})$. tert-Butyldimethylsilylchloride $(10.9 \mathrm{~g}$, $72.3 \mathrm{mmol})$ and imidazole $(9.8 \mathrm{~g}, 0.15 \mathrm{~mol})$ were added to this solution and the mixture was stirred for 18 hours at $45^{\circ} \mathrm{C}$. Water was added and the mixture was extracted with toluene. The organic solution was dried over anhydrous $\mathrm{MgSO}_{4}$, filtered and evaporated. The resulting residue was chromatographed on silica gel eluting with $40 \%$ EtOAc in hexane to yield $\mathbf{1 0}(3.98 \mathrm{~g}, 15.6 \%$ yield over two steps). 10: ESI-MS $m / z 1128(\mathrm{M}+\mathrm{H})^{+} ;[\alpha]_{\mathrm{D}}^{25}=-8.4^{\circ}\left(c 1.04, \mathrm{CHCl}_{3}\right)$; IR $v_{\max }(\mathrm{KBr}) \mathrm{cm}^{-1} 3476,2936,2859,1751,1464,1229$, $1166,1091,1055 ;{ }^{1} \mathrm{H}$ NMR $\left(500 \mathrm{MHz}, \mathrm{CDCl}_{3}\right) \delta 0.98(3 \mathrm{H}$, d, $J=6.7 \mathrm{~Hz} ; 21-\mathrm{H}), 1.73(3 \mathrm{H}, \mathrm{s} ; 22-\mathrm{H}), 1.97$ (3H, s; OAc), $2.04\left(3 \mathrm{H}, \mathrm{s}\right.$; OAc), 2.09 (3H, s; OAc), $2.33\left(6 \mathrm{H}, \mathrm{s} ; 3^{\prime}-\right.$ $\left.\mathrm{NMe}_{2}\right), 3.46$ (3H, s; 2"-OMe), 3.50 (3H, s; 3"-OMe), 3.81 $(1 \mathrm{H}, \mathrm{dd}, J=7.6,5.5 \mathrm{~Hz} ; 9-\mathrm{H}), 5.36(1 \mathrm{H}, \mathrm{d}, J=9.8 \mathrm{~Hz} ; 13-$ H), $5.90(1 \mathrm{H}, \mathrm{dd}, J=15.8,5.5 \mathrm{~Hz} ; 10-\mathrm{H}), 6.18(1 \mathrm{H}, \mathrm{d}$, 
$J=15.8 \mathrm{~Hz} ; 11-\mathrm{H})$.

\section{9-Hydro-9,20-di-O-tert-butyldimethylsilyl-2', ,4', 4"-tri-O- triethylsilyldesmycosin-3,20-acetal (11)}

Three hydroxyl groups at sugar moieties of $\mathbf{1 0}$ (3.88 g, $3.44 \mathrm{mmol}$ ) were deprotected as mentioned above and the resulting residue was dissolved in DMF $(60 \mathrm{ml})$. Triethylsilylchrolide $(3.5 \mathrm{ml}, 20.6 \mathrm{mmol})$ and imidazole $(2.8 \mathrm{~g}, 41.3 \mathrm{mmol})$ were added to this solution and the reaction mixture was stirred for 18 hours at room temperature. Water was added and the mixture was extracted with EtOAc. The organic solution was dried over anhydrous $\mathrm{MgSO}_{4}$, filtered and evaporated. The resulting residue was chromatographed on silica gel eluting with $20 \%$ EtOAc in hexane to yield $\mathbf{1 1}(3.37 \mathrm{~g}, 75.2 \%$ yield over two steps). 11: ESI-MS $m / z 1346(\mathrm{M}+\mathrm{H})^{+} ;[\alpha]_{\mathrm{D}}^{25}=-17.5^{\circ}$ (c 1.00, $\mathrm{CHCl}_{3}$ ); IR $v_{\max }(\mathrm{KBr}) \mathrm{cm}^{-1} 3436,2958,2879$, $1743,1463,1382,1251,1106,1082 ;{ }^{1} \mathrm{H}$ NMR $(500 \mathrm{MHz}$, $\left.\mathrm{CDCl}_{3}\right) \delta 1.18(3 \mathrm{H}, \mathrm{d}, J=6.1 \mathrm{~Hz} ; 21-\mathrm{H}), 1.75(3 \mathrm{H}, \mathrm{s} ; 22-$ $\mathrm{H}), 2.42\left(6 \mathrm{H}, \mathrm{s} ; 3^{\prime}-\mathrm{NMe}_{2}\right), 3.47$ (3H, s; 2"-OMe), $3.58(3 \mathrm{H}$, s; 3"-OMe), $5.44(1 \mathrm{H}, \mathrm{d}, J=10.4 \mathrm{~Hz} ; 13-\mathrm{H}), 5.84(1 \mathrm{H}, \mathrm{dd}$, $J=15.9,6.1 \mathrm{~Hz} ; 10-\mathrm{H}), 6.14(1 \mathrm{H}, \mathrm{d}, J=15.9 \mathrm{~Hz} ; 11-\mathrm{H})$.

\section{$2 \beta$-Methyl-9-hydro-9,20-di-O-tert-butyldimethylsilyl- $2^{\prime}, 4^{\prime}, 4^{\prime \prime}$-tri- $O$-triethylsilyldesmycosin-3,20-acetal (12), $2 \alpha$-Methyl-9,20-di-O-tert-butyldimethylsilyl-2', $4^{\prime}, 4^{\prime \prime}$-tri- $O$-triethylsilyldesmycosin-3,20-acetal (13)}

$n$-BuLi ( $15 \%$ solution in hexane, $1.1 \mathrm{ml}, 2.51 \mathrm{mmol})$ was added to the mixture of ${ }^{i} \operatorname{Pr}_{2} \mathrm{NEt}(0.35 \mathrm{ml}, 2.51 \mathrm{mmol})$ and THF - DMPU 2: $1(3.0 \mathrm{ml})$ at $-78^{\circ} \mathrm{C}$. After stirring for 20 minutes, $11(675.1 \mathrm{mg}, 0.502 \mathrm{mmol})$ in THF - DMPU $2: 1$ $(3.0 \mathrm{ml})$ was added to this solution. Methyl iodide $(0.16 \mathrm{ml}$, $2.51 \mathrm{mmol})$ was added and the reaction mixture was stirred for 30 minutes. The reaction mixture was added to saturated $\mathrm{NH}_{4} \mathrm{Cl}$ aq. and extracted with EtOAc. The organic solution was dried over anhydrous $\mathrm{MgSO}_{4}$, filtered and evaporated. The resulting residue was separated by silica gel pTLC (hexane-EtOAc 5:1) to yield $\mathbf{1 2}(340.1 \mathrm{mg}$, 49.8\% yield) and $\mathbf{1 3}$ (39.2 $\mathrm{mg}, 5.7 \%$ yield). 12: ESI-MS $m / z 1360(\mathrm{M}+\mathrm{H})^{+} ;[\alpha]_{\mathrm{D}}^{25}=-2.1^{\circ}\left(c \quad 0.84, \mathrm{CHCl}_{3}\right)$; IR $v_{\max }(\mathrm{KBr}) \mathrm{cm}^{-1} 3436,2957,2879,1741,1463,1381$, 1252, 1106; ${ }^{1} \mathrm{H}$ NMR $\left(500 \mathrm{MHz}, \mathrm{CDCl}_{3}\right) \delta 1.26(3 \mathrm{H}, \mathrm{d}$, $J=6.7 \mathrm{~Hz} ; 2-\mathrm{Me}), 1.69(3 \mathrm{H}, \mathrm{s} ; 22-\mathrm{H}), 2.41\left(6 \mathrm{H}, \mathrm{s} ; 3^{\prime}-\right.$ $\left.\mathrm{NMe}_{2}\right), 2.52(1 \mathrm{H}, \mathrm{dq}, J=9.2,6.7 \mathrm{~Hz} ; 2-\mathrm{H}), 3.47\left(3 \mathrm{H}, \mathrm{s} ; 2^{\prime \prime}-\right.$ $\mathrm{OMe}), 3.58$ (3H, s; 3"-OMe), $5.42(1 \mathrm{H}, \mathrm{d}, J=9.8 \mathrm{~Hz} ; 13-\mathrm{H})$, $5.71(1 \mathrm{H}, \mathrm{dd}, J=15.3,6.7 \mathrm{~Hz} ; 10-\mathrm{H}), 5.94(1 \mathrm{H}, \mathrm{d}$, $J=15.3 \mathrm{~Hz} ; 11-\mathrm{H}) ;{ }^{13} \mathrm{C}$ NMR $\left(125 \mathrm{MHz}, \mathrm{CDCl}_{3}\right) \delta 173.3$ (C-1), 43.8 (C-2), 75.9 (C-3), 15.8 (2-Me), 80.0 (C-9). 13: ESI-MS $m / z \quad 1360(\mathrm{M}+\mathrm{H})^{+} ;[\alpha]_{\mathrm{D}}^{25}=-63.6^{\circ}$ (c 0.68 , $\left.\mathrm{CHCl}_{3}\right)$; IR $v_{\text {max }}(\mathrm{KBr}) \mathrm{cm}^{-1} 3436,2958,2879,1743,1463$, 1382, 1251, 1106, 1082; ${ }^{1} \mathrm{H}$ NMR $\left(500 \mathrm{MHz}, \mathrm{CDCl}_{3}\right) \delta$
$1.81(3 \mathrm{H}, \mathrm{s} ; 22-\mathrm{H}), 2.42\left(6 \mathrm{H}, \mathrm{s} ; 3^{\prime}-\mathrm{NMe}_{2}\right), 2.46(1 \mathrm{H}, \mathrm{m} ; 2-$ $\mathrm{H}), 3.46\left(3 \mathrm{H}, \mathrm{s} ; 2^{\prime \prime}-\mathrm{OMe}\right), 3.58\left(3 \mathrm{H}, \mathrm{s} ; 3^{\prime \prime}-\mathrm{OMe}\right), 3.82(1 \mathrm{H}$, br d, $J=10.4 \mathrm{~Hz} ; 3-\mathrm{H}), 5.46(1 \mathrm{H}, \mathrm{d}, J=9.8 \mathrm{~Hz} ; 13-\mathrm{H}), 5.98$ $(1 \mathrm{H}, \mathrm{dd}, J=15.9,5.5 \mathrm{~Hz} ; 10-\mathrm{H}), 6.29(1 \mathrm{H}, \mathrm{d}, J=15.8 \mathrm{~Hz}$; $11-\mathrm{H}) ;{ }^{13} \mathrm{C}$ NMR (125 MHz, $\left.\mathrm{CDCl}_{3}\right) \delta 173.2(\mathrm{C}-1), 45.3$ (C-2), 74.1 (C-3), 13.8 (2-Me), 76.5 (C-9).

\section{$2 \beta$-Methyldesmycosin (14)}

To a solution of $12(107.3 \mathrm{mg}, 0.0789 \mathrm{mmol})$ in $\mathrm{CH}_{3} \mathrm{CN}$ $\mathrm{H}_{2} \mathrm{O} 1: 1(10 \mathrm{ml})$, difluoroacetic acid $(0.05 \mathrm{ml})$ was then added and stirred for 72 hours at $45^{\circ} \mathrm{C}$. Saturated $\mathrm{NaHCO}_{3}$ aq. was added to the reaction mixture and extracted with EtOAc. The organic solution was dried over anhydrous $\mathrm{MgSO}_{4}$, filtered and evaporated to yield a crude deprotected compound. 9-Hydroxyl group of this compound was oxidized as mentioned above. The resulting crude residue was purified by silica gel pTLC $\left(\mathrm{CHCl}_{3}-\mathrm{MeOH} 4: 1\right)$ to yield $\mathbf{1 4}$ (25.9 mg, 78.4\% yield over two steps). 14: HRTOF-MS $m / z 784.4477(\mathrm{M}-\mathrm{H})^{-}\left(\right.$calcd for $\mathrm{C}_{40} \mathrm{H}_{66} \mathrm{NO}_{14}$ : 784.4483); $[\alpha]_{\mathrm{D}}^{25}=-21.3^{\circ}\left(c 1.15, \mathrm{CHCl}_{3}\right)$; IR $v_{\max }(\mathrm{KBr})$ $\mathrm{cm}^{-1} 3450,2975,2936,1721,1595,1458,1380,1317$, 1268, 1168, 1083, 1063; ${ }^{1} \mathrm{H}$ NMR $\left(500 \mathrm{MHz}, \mathrm{CDCl}_{3}\right)$ $\delta 0.86(3 \mathrm{H}, \mathrm{t}, J=7.3 \mathrm{~Hz} ; 17-\mathrm{H}), 0.95(3 \mathrm{H}, \mathrm{d}, J=7.0 \mathrm{~Hz}$; $18-\mathrm{H}), 1.13(3 \mathrm{H}, \mathrm{d}, J=6.7 \mathrm{~Hz} ; 21-\mathrm{H}), 1.19(3 \mathrm{H}, \mathrm{d}$, $\left.J=6.4 \mathrm{~Hz} ; 6^{\prime \prime}-\mathrm{H}\right), 1.21(3 \mathrm{H}, \mathrm{d}, J=7.0 \mathrm{~Hz} ; 2-\mathrm{Me}), 1.22(3 \mathrm{H}$, d, $\left.J=6.1 \mathrm{~Hz} ; 6^{\prime}-\mathrm{H}\right), 1.72(3 \mathrm{H}, \mathrm{s} ; 22-\mathrm{H}), 2.28(1 \mathrm{H}, \mathrm{m} ; 2-\mathrm{H})$, $2.51\left(6 \mathrm{H}, \mathrm{s} ; 3^{\prime}-\mathrm{NMe}_{2}\right), 3.41$ (3H, s; 2"-OMe), $3.54(3 \mathrm{H}, \mathrm{s}$; 3"-OMe), $3.84(1 \mathrm{H}, \mathrm{m} ; 3-\mathrm{H}), 4.22\left(1 \mathrm{H}, \mathrm{d}, J=7.6 \mathrm{~Hz} ; 1^{\prime}-\mathrm{H}\right)$, $4.48\left(1 \mathrm{H}, \mathrm{d}, J=7.6 \mathrm{~Hz} ; 1^{\prime \prime}-\mathrm{H}\right), 4.88(1 \mathrm{H}, \mathrm{td}, J=9.8,2.7 \mathrm{~Hz}$; $15-\mathrm{H}), 5.82(1 \mathrm{H}, \mathrm{d}, J=10.4 \mathrm{~Hz} ; 13-\mathrm{H}), 6.15(1 \mathrm{H}, \mathrm{d}$, $J=15.8 \mathrm{~Hz} ; 10-\mathrm{H}), 7.11(1 \mathrm{H}, \mathrm{d}, J=15.8 \mathrm{~Hz} ; 11-\mathrm{H}), 9.63$ $(1 \mathrm{H}, \mathrm{s} ; 20-\mathrm{H}) ;{ }^{13} \mathrm{C}$ NMR $\left(125 \mathrm{MHz}, \mathrm{CDCl}_{3}\right) \delta 176.7$ (C-1), 43.8 (C-2), 13.7 (2-Me), 73.3 (C-3), 203.9 (C-9), 202.6 (C-20).

\section{$2 \alpha$-Methyldesmycosin (15)}

Compound $13(53.5 \mathrm{mg}, 0.0394 \mathrm{mmol})$ was deprotected in the same method applied to $\mathbf{1 2}$, followed by oxidation and purification by silica gel pTLC $\left(\mathrm{CHCl}_{3}-\mathrm{MeOH} 4: 1\right)$, yielding 15 (18.9 mg, 61.0\% yield over two steps). 15: HRTOF-MS $m / z 786.4659(\mathrm{M}+\mathrm{H})^{+}\left(\right.$calcd for $\mathrm{C}_{40} \mathrm{H}_{68} \mathrm{NO}_{14}$ : $786.4640) ;[\alpha]_{\mathrm{D}}^{25}=+3.8^{\circ}\left(c 0.43, \mathrm{CHCl}_{3}\right) ; \mathrm{IR} v_{\max }(\mathrm{KBr})$ $\mathrm{cm}^{-1} 3436,2975,2932,1722,1594,1457,1380,1267$, 1168,$1084 ;{ }^{1} \mathrm{H}$ NMR $\left(500 \mathrm{MHz}\right.$, acetone- $\left.d_{6}\right) \delta 0.94(3 \mathrm{H}, \mathrm{t}$, $J=7.3 \mathrm{~Hz} ; 17-\mathrm{H}), 0.99$ (3H, d, $J=7.3 \mathrm{~Hz} ; 2-\mathrm{Me}), 1.01(3 \mathrm{H}$, d, $J=6.7 \mathrm{~Hz} ; 18-\mathrm{H}), 1.16\left(3 \mathrm{H}, \mathrm{d}, J=6.1 \mathrm{~Hz} ; 6^{\prime \prime}-\mathrm{H}\right), 1.88$ (3H, s; 22-H), $2.48(1 \mathrm{H}, \mathrm{m} ; 2-\mathrm{H}), 2.48\left(6 \mathrm{H}, \mathrm{s} ; 3^{\prime}-\mathrm{NMe}_{2}\right)$, 3.45 (3H, s; 2"-OMe), 3.52 (3H, s; 3"-OMe), $5.88(1 \mathrm{H}, \mathrm{d}$, $J=10.4 \mathrm{~Hz} ; 13-\mathrm{H}), 6.40(1 \mathrm{H}, \mathrm{br} ; 10-\mathrm{H}), 7.23(1 \mathrm{H}, \mathrm{d}$, $J=15.3 \mathrm{~Hz} ; 11-\mathrm{H}), 9.69(1 \mathrm{H}, \mathrm{s} ; 20-\mathrm{H}) ;{ }^{13} \mathrm{C}$ NMR $(125$ MHz, acetone- $\left.d_{6}\right) \delta 177.1$ (C-1), 43.5 (C-2), 11.6 (2-Me), 
203.8 (C-9), 203.5 (C-20).

Acknowledgment We would like to thank Mrs. Y. Nozawa for LC/MS measurement. We would also like to thank Mr. T. Asaka and Dr. M. Kashimura for their helpful suggestions.

\section{References}

1. Asaka T, Manaka A, Sugiyama H. Recent development in macrolide antimicrobial research. Current Topics in Medicinal Chemistry 3: 961-989 (2003)

2. Inoue A, Deguchi T. Biosynthesis and the metabolic fate of carbon-14 labeled spiramycin I. J Antibiot 36: 442-444
(1983)

3. Kageyama S, Tsuchiya T. Selective methylations of the 2'-hydroxy and C-2 positions of 3-deoxy-5-O-(4deoxymycaminosyl)tylonolide. Carbohydrate Research 274: 269-278 (1995)

4. Cremer LC, Toth JE, Kirst HA. Synthesis and in vitro antimicrobial activity of 3-keto 16-membered macrolides derived from tylosin. J Antibiot 55: 427-436 (2002)

5. Furuuchi T, Kurihara K, Yoshida T, Ajito K. Synthesis and bbiological evaluation of novel leucomycin analogues modified at the C-3 position. J Antibiot 56: 399-414 (2003)

6. National Committee for Clinical Laboratory Standards. 1997. Methods for dilution antimicrobial susceptibility tests for bacteria that grow aerobically. NCCLS document M7-A4 